\title{
Genetic diversity in partially selfing populations with the stepping-stone structure
}

\author{
HIDENORI TACHIDA* $† \&$ HIROSHI YOSHIMARU $\ddagger$ \\ $\uparrow$ Department of Biology, Faculty of Science, Kyushu University 33, Fukuoka 812, Japan and $\ddagger$ Population Genetics \\ Laboratory, Forestry and Forest Products Research Institute, Tsukuba, Ibaraki 305, Japan
}

\begin{abstract}
A method to compute identity coefficients of two genes in the stepping-stone model with partial selfing is developed. The identity coefficients in partially selfing populations are computed from those in populations without selfing as functions of $s$ (selfing rate), $m$ (migration rate), $N$ (subpopulation size), $n$ (number of subpopulations) and $u$ (mutation rate). For small $m, 1 / N$ and $u$, it is shown that approximate formulae for the identity coefficients of two genes from different individuals are the same as those in random mating populations if we replace $N$ in the latter with $N(1-s / 2)$. Thus, the effects of selfing on genetic variability are summarized as reducing variation within subpopulations and increasing differentiation among subpopulations by reducing the subpopulation size. The extent of biparental inbreeding as measured by the genotypic correlation between truly outcrossed mates was computed in the one-dimensional stepping-stone model. The correlation was shown to be independent of the selfing rate and starts to fall off as the migration rate increases when $m N$ is larger than 0.1 .
\end{abstract}

Keywords: biparental inbreeding, fixation index, genetic variability, geographical differentiation, selfing, stepping-stone model.

\section{Introduction}

Many plant species are partially self-fertilizing (Brown, 1990; Murawski \& Hamrick, 1991, 1992; Kitamura et al., 1994). Thus, it is necessary to incorporate self-fertilization in the modelling of plant genetic diversity. Pollak (1987) computed identity coefficients of up to four genes in a partially selfing population without a geographical structure. Maruyama \& Tachida (1992) analysed the island model with partial selfing and derived formulae for identity coefficients of pairs of genes in the equilibrium state. Here, we develop a method to derive equilibrium identity coefficients of two genes in partially selfing populations with the stepping-stone structure (Kimura, 1953). It is shown that the subpopulation size is effectively reduced in partially selfing populations resulting in an increase of differentiation among subpopulations.

${ }^{*}$ Correspondence.

\section{Model analysis}

Genes are defined to be identical by descent if they have a common ancestor gene and there is no mutation in their descent from the common ancestor. We assume the selective neutrality of alleles (Kimura, 1968) and let $u$ be the mutation rate. Consider a $d$-dimensional stepping-stone model in which each subpopulation has the same size, $N$, and subpopulations are arrayed in a $d$-dimensional torus (see Maruyama, 1977). Assume that generations are discrete. For simplicity, we consider only pollen migration and the maternal gamete always comes from the same subpopulation, Let $s$ and $m_{\mathrm{p}}$ be the selfing rate and pollen migration rate, respectively, in the population. Here, in order to simplify the expressions, $s$ is defined not to include the probability of selfing which occurs randomly in random monoecious mating. There are three ways in which the paternal gamete fertilizing the maternal gamete is chosen. (1) The paternal gamete comes from the same parent as that of the maternal gamete with a 
probability of $s+\left(1-s-m_{\mathrm{p}}\right) / N$. (2) The paternal gamete comes from a different parent in the same subpopulation from that of a maternal gamete with a probability $\left(1-s-m_{p}\right)(1-1 / N)$. (3) The paternal gamete comes from a parent in one of the $2 d$ neighbouring subpopulations with a probability of $m_{\mathrm{p}}$. Under these assumptions, the subpopulations are equivalent to each other. Thus, the identity coefficient of a set of genes in the equilibrium state depends on the relative locations of the subpopulations from which the genes are sampled. For example, the inbreeding coefficient, $f$, defined as the probability of two genes in an individual being identical by descent, is the same for all individuals.

Define the following identity coefficients for pairs of genes where i.b.d. means identical by descent:

$f=\operatorname{Prob}[$ two genes in the same individual are i.b.d.];

$\theta_{0}=\operatorname{Prob}[$ two genes in different individuals from the same subpopulations are i.b.d.]; and

$\theta_{i}=$ Prob[two genes sampled from different subpopulations $i$-steps apart are i.b.d.].

Here, $i$ is a $d$-dimensional vector, the elements of which represent the numbers of steps the two subpopulations are apart in respective dimensions. In the following derivation, the identity coefficients of two genes from two different subpopulations one step apart are important. We denote the average of the $2 d$ identity coefficients for the neighbouring subpopulations by $\theta_{1}$.

Now we compare the equilibrium values of these identity coefficients. Because two uniting gametes in an individual in a subpopulation are from an individual in the same subpopulation (selfing), two different individuals in the same subpopulation, or one from the same subpopulation and the other from the neighbouring subpopulation with a probability $s+\left(1-s-m_{\mathrm{p}}\right) / N,\left(1-s-m_{\mathrm{p}}\right)(1-1 / N)$, or $m_{\mathrm{p}}$, respectively, and the probability of identity by descent is $(1+f) / 2$ if two genes come from the same individual,

$$
\begin{aligned}
f= & (1-u)^{2}\left\{\left[s+\frac{\left(1-s-m_{\mathrm{p}}\right)}{N}\right]\left(\frac{1+f}{2}\right)\right. \\
& \left.+\left(1-s-m_{\mathrm{p}}\right)\left(1-\frac{1}{N}\right) \theta_{0}+m_{\mathrm{p}} \theta_{1}\right\} .
\end{aligned}
$$

Next we consider $\theta_{0}$ for two genes in different individuals in the same subpopulation. In the following, we trace the descent of the two genes in the past and utilize the relationships between the coalescence of the genes and the identity coefficients
(Tachida, 1985; Slatkin, 1991; Slatkin \& Voelm, 1991). The time will be measured backwards in units of generations. Let $T$ be the coalescence time for the two genes. Then, $\theta_{0}$ is represented as

$\theta_{0}=\mathrm{E}\left[(1-u)^{2 T}\right]$.

This coalescence time is divided into two parts (Slatkin, 1991); $T_{1}$ when the ancestors of the two genes were in the same individual for the first time (coalescence to an individual), and $T_{2}$ for the two genes in the same individual to coalesce. Therefore,

$\theta_{0}=\mathrm{E}\left[(1-u)^{2 T_{1}}(1-u)^{2 T_{2}}\right]$.

Because $T_{1}$ and $T_{2}$ are independent, this is further simplified to

$\theta_{0}=\mathrm{E}\left[(1-u)^{2 T_{1}}\right] \mathrm{E}\left[(1-u)^{2 T_{2}}\right]$.

To compute the first term of the right-hand side of (4), we first show that the distribution of $T_{1}$ is the same as the coalescence time of two genes in a haploid population with the same population structure. Let $m$ be the gamete migration rate in the haploid population. The number of genes in each subpopulation is $N$. If we take two genes from the same subpopulation, each gene is a migrant with a probability $m$. If the two genes come from the same subpopulation (nonmigrant), the probability of the two coming from the same individual is $1 / N$. Now we consider how the two genes come from the previous generation in the selfing population. Each one of the two genes has either paternal or maternal origin with a probability one-half and is a migrant only when it has paternal origin. Thus, each gene is a migrant with a probability $m_{\mathrm{p}} / 2$. If the two genes come from the same subpopulation, the probability of the two coming from the same individual is $1 / N$. If we replace the word individual with the word gene in the above two descriptions, we can see that the probability law for the coalescence to an individual in the selfing population is the same as that of the coalescence to a gene in a haploid population which has the same geographical structure with $m_{\mathrm{p}} / 2$ replaced by $m$, and the same subpopulation size, $N$. Thus, $T_{1}$ has the same distribution as the coalescence time of two genes in the haploid population and the first term of (4) is expressed as

$\mathrm{E}\left[(1-u)^{2 T_{1}}\right]=g_{0}(u)$,

where $g_{0}(u)$ is the coancestry coefficient in the haploid population. Because the second term of the right-hand side of $(4)$ is $(1+f) / 2$, we obtain

$\theta_{0}=g_{0}(u)\left(\frac{1+f}{2}\right)$. 
The same argument can be applied to the calculation of $\theta_{i}$ which is expressed as

$\theta_{i}=g_{i}(u)\left(\frac{1+f}{2}\right)$

where $g_{i}(u)$ s are the corresponding identity coefficients for the haploid population.

Putting (6) and (7) into (1) and solving for $f$, we obtain

$f=\frac{(1-u)^{2} A\left(s, m_{\mathrm{p}}, N, \mathrm{u}\right)}{2-(1-u)^{2} A\left(s, m_{\mathrm{p}}, N, u\right)}$

with

$$
\begin{aligned}
A\left(s, m_{\mathrm{p}}, N, u\right)= & s+\left(1-s-m_{\mathrm{p}}\right)\left[\frac{1}{N}+\left(1-\frac{1}{N}\right) g_{0}(u)\right] \\
& +m_{\mathrm{p}} \mathrm{g}_{1}(u) .
\end{aligned}
$$

Other identity coefficients are obtained from $f$ using (6) and (7).

Maruyama (1977) lists explicit expressions for $g_{i}(u)$ s in the one- and two-dimensional steppingstone models. For example, in the one-dimensional stepping stone model with $n$ subpopulations, $g_{i}(u) \mathrm{s}$ for our model are expressed as

$g_{0}(u)=\frac{(1-u)^{2} w}{N+(1-u)^{2} w}$ and

$$
\begin{aligned}
g_{i}(u)= & \frac{(1-u)^{2}\left(1-g_{0}(u)\right)}{N} \sum_{k=0}^{[(n+1) / 2]} \frac{\lambda_{k}}{a_{k}\left[1-(1-u)^{2} \lambda_{k}\right]} \\
& \times \cos \frac{2 \pi i k}{n}
\end{aligned}
$$

with

$$
\begin{aligned}
& w=\sum_{k=0}^{[(n+1) / 2]} \frac{\lambda_{k}}{a_{k}\left[1-(1-u)^{2} \lambda_{k}\right]}, \\
& \lambda_{k}=\left[1-\frac{m_{\mathrm{p}}}{2}\left(1-\cos \frac{2 \pi k}{n}\right)\right]^{2}
\end{aligned}
$$

and

$a_{k}= \begin{cases}n & (k=0 \text { or } n / 2) \\ n / 2 & \text { (otherwise). }\end{cases}$
In the above expression, [i] represents the largest integer which does not exceed $i$. We can obtain the identity coefficients in the one-dimensional steppingstone model with partial selfing by putting (10) and (11) into (8), (6) and (7). Note that $w$ is not a function of $N . g_{0}(u)$ in the two-dimensional stepping-stone model is also written in the same form as that in the one-dimensional stepping-stone model with $w$ independent of $N$. In fact, this is true for the island model and a random mating population model.

In geographically structured populations such as stepping stone models, inbreeding results not only from selfing but also from mating within the local area. The latter inbreeding is called biparental inbreeding (Ennos \& Clegg, 1982). This effect is conveniently measured by the biparental genotypic correlation, $r_{\mathrm{b}}$, between truly outcrossed mates (see Waller \& Knight, 1989) defined as

$r_{\mathrm{b}}=\frac{F_{\text {out }}}{F_{\text {sel }}}$

where $F_{\text {out }}$ and $F_{\text {sel }}$ are fixation indices of a truly outcrossed and a self-fertilized individual, respectively. In terms of identity coefficients, these fixation indices are represented as

$F_{\text {out }}=\frac{\theta_{0}-\bar{\theta}}{1-\bar{\theta}}$,

$F_{\text {sel }}=\frac{(1+f) / 2-\bar{\theta}}{1-\bar{\theta}}$

where $\bar{\theta}$ is the i.b.d. probability of two genes taken from two random individuals, respectively, in the population and expressed as

$\bar{\theta}=\frac{1}{n} \sum_{i=0}^{[(n+1) / 2]} b_{i} \theta_{i}$,

$b_{i}=\left\{\begin{array}{l}1 \text { if } i=0 \text { or } n / 2 \\ 2 \text { otherwise. }\end{array}\right.$

From (6) and (7), we obtain

$r_{\mathrm{b}}=\frac{g_{0}(u)-\bar{g}(u)}{1-\bar{g}(u)}$,

where

$\bar{g}(u)=\frac{1}{n} \sum_{i=0}^{[(n+1) / 2]} b_{i} g_{i}(u)$. 


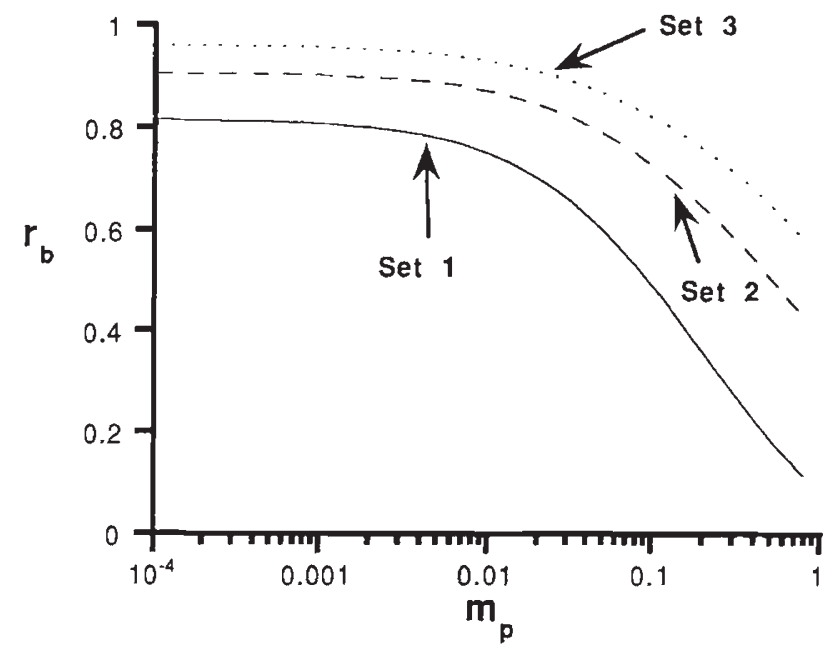

Fig. 1 The biparental genotypic correlation, $r_{b}$. Onedimensional stepping-stone model is assumed. The migration rate $m_{\mathrm{p}}$ is changed. Other parameters are $n=10$, $N=10, u=0.01$ in set $1, n=40, N=5, u=0.01$ in set 2 and $n=100, N=5, u=0.004$ in set 3 .

Because $g_{i}(u)$ s are independent of the selfing rate, this equation shows that $r_{\mathrm{b}}$ is independent of $s$. We numerically computed $r_{\mathrm{b}}$ for various $n, N, u$ changing $m_{\mathrm{p}}$ and some of the results are shown in Fig. 1. Note that although calculations are made for small $N$ and large $u$, values would be similar when $N$ and $u$ are changed keeping $N u$ constant. The genotypic correlation, $r_{\mathrm{b}}$, is a monotonic decreasing function of $m_{\mathrm{p}}$ and starts to decrease rapidly as $m_{\mathrm{p}}$ increases when $m_{\mathrm{p}} \mathrm{N}$ is larger than 0.1 . Waller \& Knight (1989) estimated $r_{\mathrm{b}}$ in Impatiens capensis and obtained values ranging from $0.1-0.4$. Such values are expected with our parameter set 1 when $m_{\mathrm{p}}$ is fairly large.

If $m_{\mathrm{p}}, u, 1 / N$ are much smaller than one and $s$, simpler expressions for $\theta_{i} \mathrm{~s}$ are possible. In this case, (8) is written as

$f=\frac{A_{\mathrm{p}}\left(s, m_{\mathrm{p}}, N, u\right)}{2-A_{\mathrm{p}}\left(s, m_{\mathrm{p}}, N, u\right)}+\mathrm{O}\left(m_{\mathrm{p}}, u, 1 / N\right)$

$A_{\mathrm{p}}\left(s, m_{\mathrm{p}}, N, u\right)=(1-s) g_{0}(u)+s$,

where $\mathrm{O}\left(m_{\mathrm{p}}, u, 1 / N\right)$ designates a function of the same order as those of $m_{\mathrm{p}}, u$ and $1 / N$. Combining the two relationships, $\theta_{0}$ is represented as

$\theta_{0}=\frac{g_{0}(u)}{2-\left[(1-s) g_{0}(u)+s\right]}+\mathrm{O}\left(m_{\mathrm{p}}, u, 1 / N\right)$.

As noted above, $g_{0}(u)$ is written as $g_{0}(u)=\frac{(1-u)^{2} w}{N+(1-u)^{2} w}$

Substituting this into (18), with some rearrangement, we obtain

$\theta_{0}=\frac{(1-u)^{2} w}{2 N(1-s / 2)+(1-u)^{2} w}+\mathrm{O}\left(m_{\mathrm{p}}, u, 1 / N\right)$.

This formula shows that $\theta_{0}$ for a partially selfing population is computed by regarding $N(1-s / 2)$ as the effective size of the subpopulation and using the formula for the corresponding diploid population without selfing. This relationship was noted by Golding \& Strobeck (1980), and Pollak (1987) for populations without any geographical structures and by Maruyama \& Tachida (1992) for the island model.

We can show that this relationship also holds for other identity coefficients, $\theta_{i}$, if $m_{\mathrm{p}}, u, 1 / N$ are much smaller than one, considering the coalescence of the two genes in different subpopulations (Slatkin, 1991). Let $T_{\mathrm{b}}$ be the time when the ancestors of the two genes were in the same subpopulation for the first time and define $T_{\mathrm{w}}=T-T_{\mathrm{b}}$ where $T$ is the total coalescence time of the two genes as in the previous argument. Then, we can write $\theta_{i}$ as

$\theta_{i}=\mathrm{E}\left[(1-u)^{2 T_{\mathrm{b}}}\right] \mathrm{E}\left[(1-u)^{2 T_{\mathrm{w}}}\right]$.

Because the first ancestor genes which enter the same subpopulation are in the same individual with a probability $1 / N$, the equation is expressed as

$$
\begin{aligned}
\theta_{i} & =\mathrm{E}\left[(1-u)^{2 T_{\mathrm{b}}}\right] \frac{1}{N}\left[\frac{1+f}{2}+(N-1) \theta_{0}\right] \\
& =\mathrm{E}\left[(1-u)^{2 T_{\mathrm{b}}}\right] \theta_{0}+\mathrm{O}(1 / N) .
\end{aligned}
$$

Note that because $T_{\mathrm{b}}$ does not depend on the size, $N$, of the subpopulation, $\mathrm{E}\left[(1-u)^{2 T_{b}}\right]$ does not depend on it. This means that $N$ enters into the formula only through $\theta_{0}$ and thus we can compute $\theta_{i}$ by regarding $N(1-s / 2)$ as the effective size of the subpopulation.

How good is this approximation of regarding $N(1-s / 2)$ as the effective size of the subpopulation? To check this, we numerically computed $\theta_{0}$ for various values of $N, m_{\mathrm{p}}, n$ and some of the results are shown in Fig. 2. The discrepancies between the exact and approximate values are very small even for the small $N$ used in the computations. Even for fairly large $m_{\mathrm{p}}$, the difference is less than a few per cent. Thus, the approximation works very well for most parameter ranges encountered in nature.

(C) The Genetical Society of Great Britain, Heredity, 77, 469-475. 


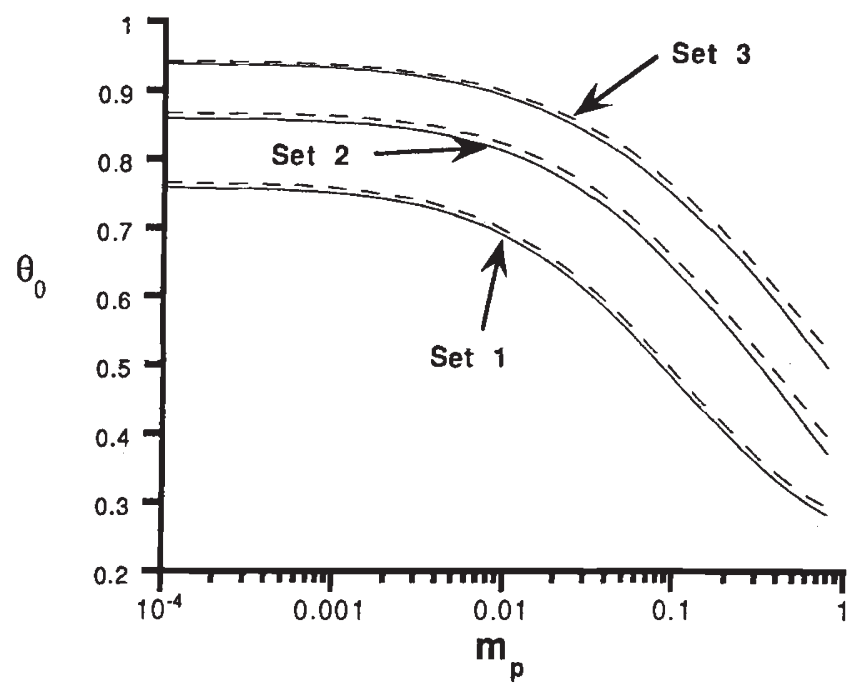

Fig. 2. The exact and approximate values of the identity coefficient, $\theta_{0}$, of two genes in different individuals in the same subpopulation as functions of $m_{\mathrm{p}}$ assuming the one-dimensional stepping-stone model. The straight lines are computed using the exact solution (6) with (8).

Broken lines are computed using the approximate solution (20). Other parameters are $n=10, N=10, u=0.01$, $s=0.5$ in set $1, n=40, N=5, u=0.01, s=0.5$ in set 2 and $n=100, N=5, u=0.004, s=0.5$ in set 3 .

\section{Discussion}

In the present paper, we developed a method to compute identity coefficients in stepping-stone models with partial selfing from those without selfing. Because exact solutions are available for stepping-stone models (Maruyama, 1977), we could use those solutions to obtain the exact identity coefficients in partially selfing populations. Although we assumed stepping-stone models in the derivation, the critical assumption is that $f$ is constant over the subpopulations. If this holds true, (7) holds and we can compute the identity coefficients in the partially selfing population in the same way.

For small $m_{\mathrm{p}}, u, 1 / N$, we showed that $N(1-s / 2)$ can be used as the effective size of the subpopulation. This relationship can be derived also by the following intuitive argument. Assume that we sampled two genes from two different individuals in the same subpopulation. We consider the probability of the two genes becoming identical by descent in the parental generation. The two genes come from the same individual with a probability $1 / N$ if we ignore the higher order terms of $m_{\mathrm{p}}$ and $1 / N$. Once the two genes are in an individual, they coalesce soon without going out of an individual (the coalescence in an individual) and become identical by descent with a probability $s /(2-s)$. Therefore, if we ignore mutation during the coalescence in an individual, two random genes in one individual are identical by descent with a probability $[1+s /(2-s)] / 2=1 /(2-s)$. If we regard this event of quickly becoming identical by descent as having occurred in the parental generation, the probability of the two parental genes being identical by descent is

$\frac{1}{2-s} \frac{1}{N}=\frac{1}{2 N(1-s / 2)}$.

This shows that $N(1-s / 2)$ can be used as an effective number for partially selfing populations. Our derivation in the previous section shows that this rough argument is actually applicable as an approximation. Note that this argument does not rely on a specific geographical structure of the population. Thus, the method of using $N(1-s / 2)$ as the effective size seems not to be restricted to symmetrical structures such as the island and stepping-stone models and might be used, for example, for the cases where parameters such as the selfing rate and subpopulation size change among subpopulations (Karron et al., 1995).

In our computation, only pollen migration was considered. This argument goes similarly if there is only seed migration. Let $m_{\mathrm{s}}$ be the seed migration rate. For the coefficient, $f$, for the two genes in the same individual, the following equation holds in the equilibrium state:

$f=(1-u)^{2}\left[\left(s+\frac{1-s}{N}\right) \frac{1+f}{2}+(1-s)\left(1-\frac{1}{N}\right) \theta_{0}\right]$.

For two genes in different individuals, there are three cases with regard to whether they are migrants or not. (1) Both are nonmigrant with a probability $\left(1-m_{s}\right)^{2}$. (2) One is migrant and the other is nonmigrant with a probability $2\left(1-m_{\mathrm{s}}\right) m_{\mathrm{s}}$. (3) Both are migrants with a probability $m_{\mathrm{s}}^{2}$. Therefore, $\theta_{i}$ s are expressed as in (7) but in this case we use $m_{s}$ as the migration rate to compute $g_{i}(u)$ in the corresponding haploid population. If $u, m_{\mathrm{s}}, 1 / N$ are much smaller than one, we obtain the same approximation as that in the case of pollen migration in which $(1-s / 2) N$ can be used as the effective size of the subpopulation.

On the other hand, if both seed and pollen migration occur, we can not just use $g_{i}(u)$ computed from the corresponding haploid population to compute the exact solutions in partially selfing populations. For example, a gene may come from a subpopulation two steps apart from the subpopulation in 
which it resides because the two types of migration can occur for a gene in one generation. This never happens in the corresponding haploid population. However, if $m_{\mathrm{s}}, m_{\mathrm{p}}, u, 1 / N$ are much smaller than one, the probability of such two-step migration is very small and such events can be ignored. Then, we can use $(1-s / 2) N$ as the effective number and compute the identity coefficients from the corresponding haploid population with the migration rate $m_{\mathrm{s}}+m_{\mathrm{p}} / 2$ as noted by Maruyama \& Tachida (1992).

From the above argurments, we can summarize the general effect of partial selfing on identities of two genes in different individuals as replacing the subpopulation size, $N$, in populations without selfing with $N(1-s / 2)$. This reduction of the subpopulation size reduces the variation within subpopulations and promotes differentiation among subpopulations.

Caballero \& Hill (1992) derived a general formula to compute the variance effective number of nonrandom mating populations. For a Poisson distribution of family size and a specified system of partial inbreeding, the effective size is represented as $N_{\mathrm{e}}=N /\left(1+F_{\text {IS }}\right)$ where $F_{\text {IS }}$ is Wright's $F_{\text {Is }}$ statistic. If we substitute the equilibrium inbreeding coefficient, $s /(2-s)$, for infinite partially selfing populations (see, for example, Hedrick \& Cockerham, 1986) into $F_{\text {IS }}$, we obtain $N_{\mathrm{e}}=N(1-s / 2)$. Thus, we can also reach the same conclusion using the argument of Caballero \& Hill (1992). However, there are two merits in our approach. First, the variance effective number is obtained by considering the change of the variance of the gene frequency whereas our approach directly computes the equilibrium identity coefficients under the pressure of mutation. Therefore, our approach is conceptually more direct. Secondly, our approach is based on the exact calculation of Maruyama (1977) and as long as our concern is the identity coefficients, the result is exact. If we ignore the second-order terms of $u, m$, and $1 / N$, the two approaches will give the same second moments of gene frequencies with $(1-s / 2) N$ as an effective size of a subpopulation. However, if some second-order terms cannot be neglected, there will be difference although it might not be so large (see Fig. 2). Thus, our approach, for example, can provide the condition for the applicability of this simple relationship, $N_{\mathrm{e}}=(1-s / 2) N$, in selfing populations.

Finally, our formula can be used to compute the probability distribution of the number, $k$, of different sites between two sequences under the infinite site model without recombination (Watterson, 1975). Griffiths (1981) and Tachida (1985) showed that the generating function, $\Theta(z)$, of $k$ is related to the corresponding identity coefficient, $\theta(u)$, by

$\Theta(z)=\theta(u(1-z))$.

Thus, for example, the expected number of differences is

$\mathrm{E}[k]=\Theta^{\prime}(0)$.

This formula can be used to assess the magnitude of nucleotide diversity ( $\mathrm{Nei} \& \mathrm{Li}, 1979$ ) in partially selfing populations for which DNA data are accumulating (Clegg, 1990). Also, once $\mathrm{E}[k]$ is computed, we can compute the expected squared difference, $\mathrm{E}[S]$, of repeat numbers between two genes in microsatellite loci assuming the stepwise mutation model (Ohta \& Kimura, 1973). Utilizing the argument described in Garza et al. (1995), we obtain $\mathrm{E}[S]$ in terms of $\mathrm{E}[k]$ as

$\mathrm{E}[S]=\mathrm{E}[k] \sigma_{\mathrm{m}}^{2}$,

where $\sigma_{\mathrm{m}}^{2}$ is the variance of the change in allele size given that a mutation occurs. Now microsatellite loci are found in a wide variety of plant species (e.g. Terauchi, 1994; Yang et al., 1994; Roder et al., 1995) and they can be used to investigate microgeographical genetic structures and mating systems of plant populations.

\section{Acknowledgements}

We thank two anonymous reviewers for comments on the manuscript. This research was partially supported by NIG Cooperative Research Programme, a grant from the Society for the Promotion of Genetics and a grant-in-aid from the Ministry of Education, Science and Culture of Japan.

\section{References}

BRown, A. H. D. 1990. Genetic characterization of plant mating systems. In: Brown A. H. D., Clegg, M. T., Kahler, A. L. and Weir, B. S. (eds) Plant Population Genetics, Breeding, and Genetic Resources, pp. 145-162. Sinauer Associates, Sunderland, MA.

CABAllero, A. AND Hill, w. G. 1992. Effective size of nonrandom mating populations. Genetics, 130, 909-916.

ClEGG, M. T. 1990. Molecular diversity in plant populations, In: Brown, A. H. D., Clegg, M. T., Kahler, A. L. and Weir, B. S. (eds) Plant Population Genetics, Breeding, and Genetic Resources, pp. 98-115. Sinauer Associates, Sunderland, MA.

ENNOS, R. A. AND CLEGG, M. T. 1982. Effect of population substructuring on estimates of outcrossing rate in plant populations. Heredity, 48, 283-292.

GARZA, J. C., SLATKiN, M. AND FREIMER, N. B. 1995. Microsatellite allele frequencies in humans and chimpanzees, 
with implications for constraints on allele size. Mol. Biol. Evol., 12, 594-603.

GOLDING, G. B. AND STROBECK, C. 1980. Linkage disequilibrium in a finite population that is partially selfing. Genetics, 94, 777-789.

GRIFFITHS, R. C. 1981. The number of heterozygous loci between two randomly chosen completely linked sequence of loci in two subdivided population models. J. Math. Biol., 12, 251-261.

HEDRICK, P. W. AND COCKERHAM, C. C. 1986. Partial inbreeding: equilibrium heterozygosity and the heterozygosity paradox. Evolution, 40, 856-861.

KARRON, J. D., THUMSER, N. N., TUCKER, R. AND HESSENAUER, A. J. 1995. The influence of population density on outcrossing rates in Mimulus ringens. Heredity, 75, $175-180$.

KIMURA, M. 1953. "Stepping stone" model of population. Ann. Rep. Nat. Inst. Genet., 3, 63-65.

KIMURA, M. 1968. Evolutionary rate at the molecular level. Nature, 217, 624-626.

KITAMURA, K., RAHMAN, M. Y. B. A., OCHIAI, Y. AND YOSHIMARU, H. 1994. Estimation of the outcrossing rate on Dryobalanops aromatica Gaertn. f. in primary and secondary forests in Brunei, Borneo, Southeast Asia. Pl. Sp. Biol., 9, 37-41.

MARUYAMA, K. AND TACHIDA, H. 1992. Genetic variability and geographical structure in partially selfing populations. Jap. J. Genet., 67, 39-51.

maruyama, T. 1977. Stochastic Problems in Population Genetics. Springer, Berlin.

MURAWSKI, D. A. AND HAMRICK, J. L. 1991. The effect of the density of flowering individuals on the mating systems of nine tropical tree species. Heredity, 67, 167-174.

MURAWSKI, D. A. AND HAMRICK, J. L. 1992. The mating system of Cavanillesia platanifolia under extremes of flowering-tree density: a test of predictions. Biotropica,
24, 99-101.

NEI, M. AND LI, W.-H. 1979. Mathematical model for studying genetic variation in terms of restriction endonucleases. Proc. Natl. Acad. Sci. U.S.A., 76, 5269-5273.

OHTA, T. AND KIMURA, M. 1973. A model of mutation appropriate to estimate the number of electrophoretically detectable alleles in a finite population. Genet. Res., 22, 201-204.

POLLAK, E. 1987. On the theory of partially inbreeding finite populations. I. Partial selfing. Genetics, 117, 353-360.

RÖDER, M. S., PLASCHKE, J., KÖNIG, S. U., BÖRNER, A., SORRELS, M. E., TANKSKEY, S. D. AND GANAL, M. W. 1994. Abundance, variability and chromosomal location of microsatellites in wheat. Mol. Gen. Genet., 246, 327-333.

SLATKIN, M. 1991. Inbreeding coefficients and coalescence times. Genet. Res., 58, 167-175.

SLATKIN, M. AND VOELM, L. 1991. $F_{\text {ST }}$ in a hierarchical island model. Genetics, 127, 627-629.

TACHIDA, H. 1985. Joint frequencies of alleles determined by separate formulations for mating and mutation systems. Genetics, 111, 963-974.

TERAUCHI, R. 1994. A polymorphic microsatellite marker from the tropical tree Dryobalanops lanceolata (Dipterocarpaceae). Jap. J. Genet., 69, 567-576.

WALLER, D. M. AND KNIGHT, S. E. 1989. Genetic consequences of outcrossing in the cleistogamous annual, Impatiens capensis. II. Outcrossing rates and genotypic correlations. Evolution, 43, 860-869.

WATTERSON, G. A. 1975. On the number of segregating sites in genetical models without recombination. Theor. Pop. Biol., 7, 256-276.

YANG, G. P., SAGHAI MAROOF, M. A., XU, C. G., ZHANG, Q. AND BIYASHEV, R. M. 1994. Comparative analysis of microsatellite DNA polymorphism in landraces and cultivars of rice. Mol. Gen. Genet., 245, 187-194. 